\title{
The Calculation of Electric Power Flow Using Neural Network Systems
}

\author{
Member Masanori Sugisaka (Oita University) \\ Non-member Kyoichi Murakami (Oita University) \\ Member Fumio Ishikawa (Chyugoku Electric Power Co., Inc.) \\ Member Hiroshi Shibuya (Chyugoku Electric Power Co., Inc.) \\ Member Minoru Kitagawa (Chyugoku Electric Power Co., Inc.)
}

\begin{abstract}
The neural network system employed in this study has 3-layers structure which is composed of an input, a hidden, and an output layers. As a simulation example the calculation of electric power flow was considered. The results in this study show an idea how to construct the neural network system for fast calculation or simulation of the electric power equations.
\end{abstract}

Key words : Load flow calculation, Artificial neural network system, Direct current method

\section{Introduction}

The artificial neural network system ${ }^{(1)-(5)}$ is a mathematical model which simulates neural networks in human brain. The brain has many superior features such as parallel processing of information, learning function, self-organizing ability, etc. Therefore, it is expected that the neural network system obtained by modeling the brain has similar superior features as it has and could easily solve the problems which are difficult to be calculated by Neuman-type computers.

The neural network system, which is a nonlinear learning system, has an advantage that it is able to calculate the load flow states under both stable and unstable conditions due to heavy loads or sudden disturbances.

In this study the neural network system with the superior parallel processing property in addition to the above property is used for the load flow calculation. The neural network system is learned by the calculated values of load flow at each node and thereafter can calculate various load flow states in the power system. The analysis of the simulation study is given.

\section{Application of neural network system for load flow calculation}

\subsection{Learning algorithms ${ }^{(1)}$}

In this study, we construct multi-input and multi-output units in a layer and assume that the connections in each layer and the connections from the output layer to the input layer do not exist in the neural network system. This neural network system is learned by the following three learning algorithms.

(i) Sequential modified method (back propergation method)

This method modifies the synapsis weights and offsets at each acceptance of learning patterns where the sigmoid function given by

$$
f(x)=1 /\left\{1+\exp \left(-2 x / u_{0}\right)\right\}
$$

is used as the response of unit.

(ii) Moment method

This method modifies the synapsis weights and offsets by considering the contribution from the errors in the output layer. The current modification $\Delta W(t)$ is calculated from the sum of both the former modification $\Delta W(t-1)$ and the modification from the error $d$ such that

$$
\Delta W(t)=d+m \Delta W(t-1)
$$

where $d$ is $\partial E / \partial W$ [refer to Ref. (1)], $E$ is the errors between the outputs of the neural network system and the teaching signals, and $m$ is moment coefficient. In the simulation $m=0.9$ and $\Delta W(0)=$ randam value were used.

(iii) Modified moment method

This method modifies the synapsis weights and offsets in various directions and after the learning process 
proceeds modifies them in the direction which is near to the former modification. The algorithm is given by

$$
\begin{aligned}
& \Delta W(t)=d+m(t) \Delta W(t-1) \cdots \cdots \cdots \cdots \cdots \cdots \cdots(3) \\
& m(t)=m(t-1)+\Delta m \cdots \cdots \cdots \cdots \cdots \cdots \cdots \cdots \cdots \cdots(4)
\end{aligned}
$$

where $m(0)=0.6$ and $\Delta m=0.02$ were used.

\subsection{Power system to be learned}

In information processing the learning function of the neural network system does not require the strict mathematical model of the object to be processed and but requires only appropriate data which are available.

In this study the active power $P_{i}$ and phase angle $\delta_{i}$ of node $i$ calculated from the direct current method, which is an approximate load flow calculation method, are used as the data for the object to be learned. Namely, the active powers and phase angles are used as the inputs and teaching signals for the neural network system, respectively. In the neural network system only the value ranged from 0 to 1 can be learned and therefore the power unit is employed in order to express the various values in the power system.

The power system in problem 6.4 on page 186 in Ref. (6) is employed as the model of power system used for learning which is shown in Fig. 1. The values of reactance for each line are the same as in problem 6.4. For this system model we consider the convergence condi. tions when both the parameter $u_{0}$ in the sigmoid function and number of the units in the hidden layer are

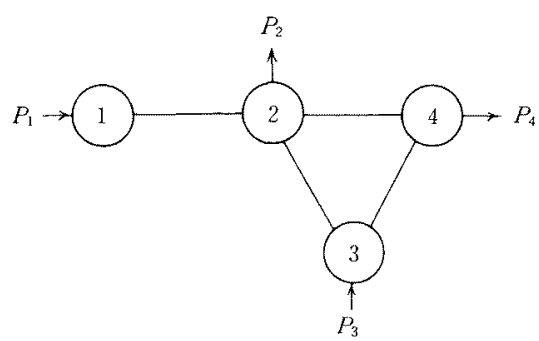

Fig. 1. Model of electric power system with 4 nodes.

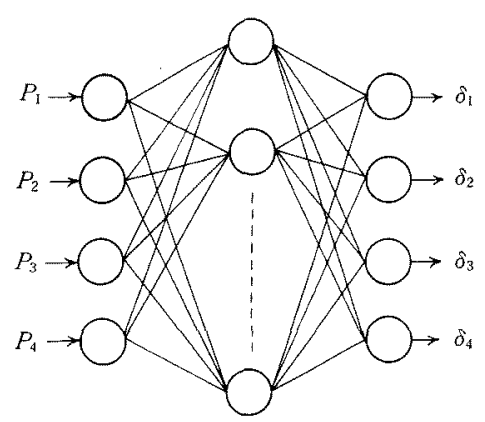

Fig. 2. Three layers neural network system. changed in the 3-layers neural network system shown in Fig. 2.

\section{Simulation study}

In the simulation study on the learning of the neural network system the number of the units in the hidden layer is changed as 10,20 and 30 and also the parameter of the sigmoid function $u_{0}$ is changed as $0.75,2.0,3.0$, and 4.0. The number of the data used for the learning is 30 pairs which is generated ramdomly from the $P-\delta$ system in the problem 6.4 in Ref. (6). As the criterion of the convergence the sum of the mean square error (denoted MSE) between the teaching signals and the phase angles of the output layer is used. If the sum is less than $1.5 E-5$, the learning is regarded as completed.

In Table 1 the average numbers of learning for three learning methods stated in Section 2.1 are illustrated. In Figs $3 \sim 5$ the convergence properties of the modified moment method are illustrated when both the parameter $u_{0}$ and the number of units in the hidden layer are changed as 10,20 , and 30 .

In Table 1 notation ( - ) means that the number of the learning exceeded 3,000 in one of the neural network systems with three types of units $(10,20,30)$ in the hidden layer.

From this table it is seen that the modified moment method is the most effective method compared with the other two methods. Moreover, although the modified moment method with $u_{0}=3.0$ seems to be best among

Table 1. Average numbers of learning for three learning methods.

\begin{tabular}{c|c|c|c}
\hline$u_{0}$ & $(\mathrm{i})$ & (ii) & (iii) \\
\hline 0.75 & - & - & - \\
\hline 2.0 & $1,854.3$ & 993.7 & 845.3 \\
\hline 3.0 & - & $2,289.3$ & 741.7 \\
\hline 4.0 & - & - & 846.3 \\
\hline
\end{tabular}

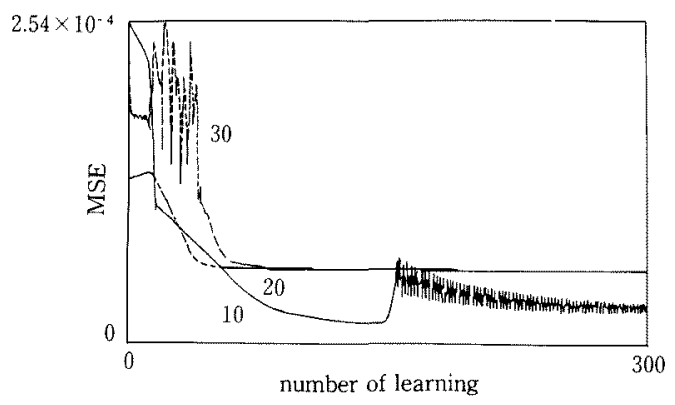

Fig. 3. Convergence process of modified moment learning method with $u_{0}=2.0$. 


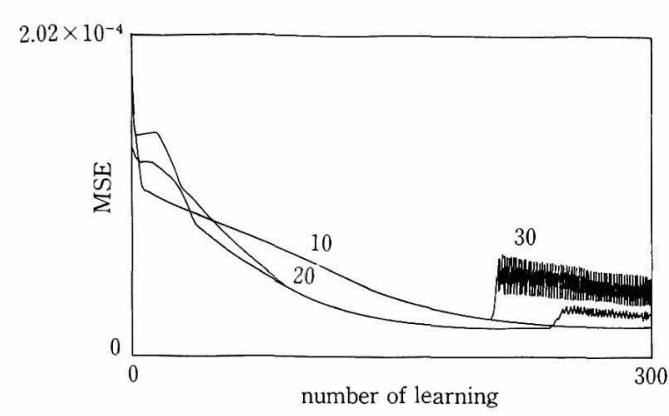

Fig. 4. Convergence process of modified moment learning method with $u_{0}=3.0$.

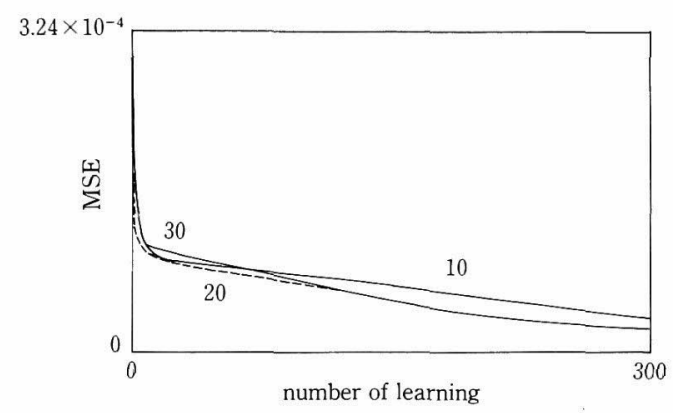

Fig. 5. Convergence process of modified moment learning method with $u_{0}=4$.

the others the convergence process of the method for the neural network system with 30 units in the hidden layer is oscillatory as seen from Fig. 4 and hence the number of the learning is large. Also as seen from Fig. 3 the convergence process for the neural network system with 10 units in the hidden layer is oscillatory. Consequently, it is concluded that the best learning method for the load flow calculation of the power system model is the modified moment method with $u_{0}=4.0$.

\section{Conclusions}

In this simulation study we used the 3-layers neural network system for the the load flow calculation in the power system model with 4 nodes. We discussed the effects of both the 3 different learning algorithms and the parameter $u_{0}$ of the sigmoid function on the convergence processes. It was seen from the simulation that there exsists an appropriate learning method and a parameter of the sigmoid function for the load flow calculation.

The future studies are on the learning of the neural network system to calculate the load flows for the power system with the large number of nodes and on the developement of the neural network system for the load flow calculation in large scale power systems. After those studies have been completed the neural network system for the load flow calculation which is learned using the measured values from a real power system could be in practical use. In order to achieve the practical use of the neural network system for the load flow calculation further studies are necessary to overcome the difficulties stated above such as selection of the number of units in the hidden layer, the number of the nodes in the power systems to be handled, and the determination of appropriate parameters. Also in order to achieve the fast calculation of the power flows under the disturbances the application of neurocomputers or neuro hardwares is inevitably necessary and this study is now in our investigations.

(Manuscript received Dec., 24, '93, revised April 4, '94)

\section{References}

(1) K. Nakano, K. Iinuma, Nyuronnettogurupu, \& S. Kiritani : Nyuumon to Zisyuu Nyuroconpyuta (Neuro computer) (in Japanese), p. 28 (1989) Gijyutsuhyouronsha

(2) K. Kyuma \& T. Nakayama: Nyuroconpyutakougaku (Neuro Computer Engineering) (in Japanese), p. 184 (1992) Kougyouchousakai

(3) T. Kikuchi : Nyuumonnyuroconpyuta (in Japanese), p. 59 (1990) Oomusha

(4) R. P. Lippmann: "An Introduction to Computing with Neural Nets", IEEE Magazine, p. 4 (1987-4) (or in Artificial Neural Networks: Theoretical Concepts edited by V. Vemuri, 1988)

(5) M. Sugisaka \& M. Teshnelab: "Fast Pattern Recognition by Using Moment Invariants Computation via Artificial Neural Networks", Control Theory \& Advanced Technology, 9, No. 4 (1993)

(6) K. Aratame: Denryokukeitougijutsukeisan no kiso (in Japanese), p. 155 (1980) Denkishoin

Masanori Sugisaka (Member) He was born in Taiwan,

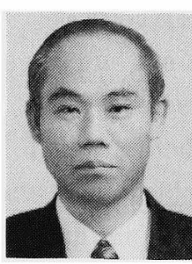
Japan in 1944. He graduated from the Doctor Course in the Department of Electrical Engineering of Kyushu University, Fukuoka, Japan in 1973 and received the Doctor of Engineering Degree in Electrical Engineering from the same University in 1975 . He is now a Professor of the Department of Electrical and Electronic Engineering, Oita University, Oita, Japan. During the 1980 1981 academic year, he was a visiting professor at Drexel University, Philadelphia, U.S.A. His interests are in the development of neuro-computing technologies for visual tracking systems, fuzzy controls of robots with artificial brains based on neuro-computers in spaces, etc.

Kyouichi Murakami (Non-member) (Photograph will be omitted)

He graduated from the Master Course in the Department of Engineering of Oita University, Oita, Japan in 1994 and works now in Kyushu Electric Power Co., Inc. 
Fumio Ishikawa (Member) He was born on February 8, $=1$
-1 1960. He received B.S. and M.S. degrees in Electric Engineering from Yokohama National University in 1984. Since 1988 he has engaged in research on analysis of power systems at Technical Research Center of Chugoku Electric Power Co., Inc.

Hiroshi Shibuya (Member) He was born on January 1, 1944. 9 He received the B.S. degree in Phisics from Shimane University in 1967. In the same year he joined the Chugoku Electric Power Co., Inc. Since 1990 he has engaged in research on analysis of power systems. Currently, he is manager of Electric Engineering Division of Technical Research Center.
Minoru Kitagawa (Member) He was born on January 6,

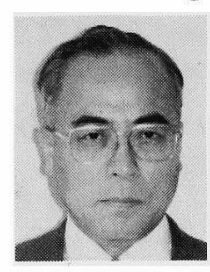
1937. He graduated from Department of Electric Engineering at Ube Industrial High School. In same year he joined the Chugoku Electrinc Power Co., Inc. He received the Ph. D. degree from Tokyo University, Since 1965 he has been engaged in research on power systems at the Technical Research Center. Currently, he is General Manager of the Technical Research Center.

$\& \infty \quad \& \infty$

